\title{
Intensifying Electron Utilization by Surface-Anchored Rh Complex for Enhanced Nicotinamide Cofactor Regeneration and Photoenzymatic $\mathrm{CO}_{2}$ Reduction
}

\author{
Yuqing Cheng, ${ }^{1,2}$ Jiafu Shi $\mathbb{D}^{2,3,4}$ Yizhou Wu, ${ }^{1,2}$ Xueying Wang, ${ }^{3}$ Yiying Sun, ${ }^{1,2}$ Ziyi Cai, ${ }^{1,2}$ \\ Yu Chen, ${ }^{3}$ and Zhongyi Jiang ${ }^{1,2}$ \\ ${ }^{1}$ Key Laboratory for Green Chemical Technology of Ministry of Education, School of Chemical Engineering and Technology, \\ Tianjin University, 92 Weijin Road, Nankai District, Tianjin 300072, China \\ ${ }^{2}$ Collaborative Innovation Center of Chemical Science and Engineering (Tianjin), 92 Weijin Road, Nankai District, \\ Tianjin 300072, China \\ ${ }^{3}$ School of Environmental Science \& Engineering, Tianjin University, 92 Weijin Road, Nankai District, Tianjin 300072, China \\ ${ }^{4}$ State Key Laboratory of Biochemical Engineering, Institute of Process Engineering, Chinese Academy of Sciences, \\ Beijing 10090, China
}

Correspondence should be addressed to Jiafu Shi; shijiafu@tju.edu.cn and Zhongyi Jiang; zhyjiang@tju.edu.cn

Received 21 October 2020; Accepted 27 December 2020; Published 18 February 2021

Copyright (C) 2021 Yuqing Cheng et al. Exclusive Licensee Science and Technology Review Publishing House. Distributed under a Creative Commons Attribution License (CC BY 4.0).

\begin{abstract}
Solar-driven photocatalytic regeneration of cofactors, including reduced nicotinamide adenine dinucleotide (NADH), reduced nicotinamide adenine dinucleotide phosphate $(\mathrm{NADPH})$, and reduced flavin adenine dinucleotide $\left(\mathrm{FADH}_{2}\right)$, could ensure the sustainable energy supply of enzymatic reactions catalyzed by oxidoreductases for the efficient synthesis of chemicals. However, the elevation of cofactor regeneration efficiency is severely hindered by the inefficient utilization of electrons transferred on the surface of photocatalysts. Inspired by the phenomenon of ferredoxin-NADP ${ }^{+}$reductase (FNR) anchoring on thylakoid membrane, herein, a homogeneous catalyst of rhodium $(\mathrm{Rh})$ complex, $\left[\mathrm{Cp} * \mathrm{Rh}(\mathrm{bpy}) \mathrm{H}_{2} \mathrm{O}\right]^{2+}$, was anchored on polymeric carbon nitride (PCN) mediated by a tannic acid/polyethyleneimine (TA/PEI) adhesive layer, acquiring PCN@TA/PEI-Rh core@shell photocatalyst. Illuminated by visible light, electrons were excited from the PCN core, then transferred through the TA/PEI shell, and finally captured by the surface-anchored Rh for instant utilization during the regeneration of NADH. The TA/PEI-Rh shell could facilitate the electron transfer from the PCN core and, more importantly, achieved $\sim 1.3$-fold elevation of electron utilization efficiency compared with PCN. Accordingly, the PCN@TA/PEI-Rh afforded the NADH regeneration efficiency of $37.8 \%$ after $20 \mathrm{~min}$ reaction under LED light $(405 \mathrm{~nm})$ illumination, over 1.5 times higher than PCN with free Rh. Coupling of the NADH regeneration system with formate dehydrogenase achieved continuous production of formate from carbon dioxide $\left(\mathrm{CO}_{2}\right)$. Our study may provide a generic and effective strategy to elevate the catalytic efficiency of a photocatalyst through intensifying the electron utilization.
\end{abstract}

\section{Introduction}

In living organisms, enzymes are the "catalytic machine" to trigger biological reactions for ensuring the steady implementation of metabolic processes. The features of such "catalytic machine" combined with the reactivity of synthetic chemical catalyst have spawned the field of chemoenzymatic catalysis, which can be categorized into three reaction types, i.e., sequential reactions, concurrent reactions, and cooperative reactions [1].
As a typical cooperative chemoenzymatic catalysis, photoenzymatic catalysis inherits the light harvesting capability of semiconductor photocatalyst and high activity/selectivity of enzyme, which can convert ubiquitous and clean solar energy into chemical energy. Till now, the photoenzymatic catalysis has been applied for carbon dioxide $\left(\mathrm{CO}_{2}\right)$ reduction [2-4], hydrogen/oxygen $\left(\mathrm{H}_{2} / \mathrm{O}_{2}\right)$ evolution [5-7], and biomass conversion $[8,9]$. During the photoenzymatic process, the nicotinamide cofactor is predominantly used as "energy currency" to coordinate photocatalysis and 


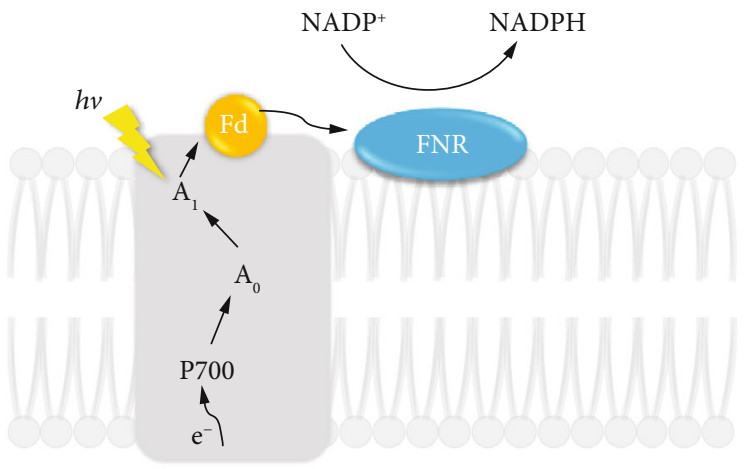

Photosystem I

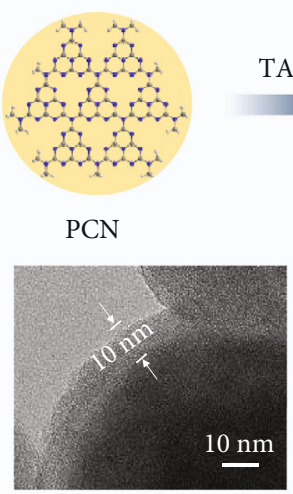

(a)
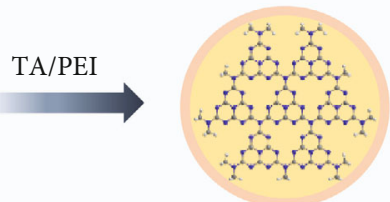

PCN@TA/PEI

PCN

TA/PEI

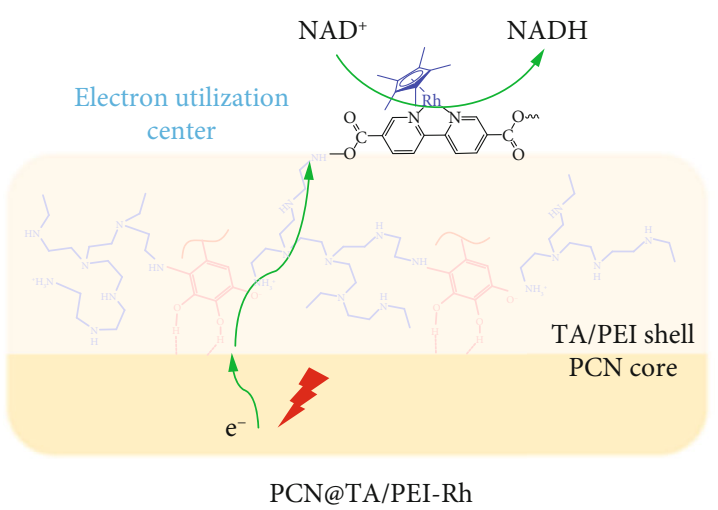

(b)

FIgURE 1: (a) Schematic diagram of NADPH regeneration in natural photosynthesis. (b) Schematic diagram of photocatalytic NADH regeneration with PCN@TA/PEI-Rh. (c) Schematic preparation of PCN@TA/PEI-Rh core@shell photocatalyst (the bottom left part was the TEM image of $\left.\mathrm{SiO}_{2} @ \mathrm{TA} / \mathrm{PEI}\right)$.

enzyme catalysis. Meanwhile, the photocatalytic regeneration of nicotinamide cofactor usually exhibits much lower rate $\left(10^{-3}-10^{-2} \mathrm{~s}^{-1}\right)$ compared with the enzymatic reaction $\left(10^{2}\right.$ $10^{3} \mathrm{~s}^{-1}$ ) [10]. Design and preparation of high-performance photocatalyst materials is thus urgently required for acquiring an improved nicotinamide cofactor regeneration efficiency.

In general, the photocatalytic process includes three main steps: charge generation, charge transfer, and charge utilization [11]. The excitation of photocatalyst by light generates holes and electrons. Usually, only part excited electrons are transferred to surface of photocatalyst and then utilized by the catalytic center to trigger the reduction reaction. To elevate the photocatalytic efficiency, the intensification of electron generation and/or electron transfer has been actively explored, which could be realized by engineering the regular structure [12], increasing the specific surface area $[13,14]$, optimizing the conduction interface [15], designing heterojunctions [16-18], and so on. Albeit grand achievements in the elevation of either electron generation or electron transfer, the intensification of electron utilization is rarely reported [19]. For a photocatalytic reaction, particularly photocatalytic nicotinamide cofactor regeneration, the electron utilization is severely hindered by the longer electron transfer and lower contact probability between the photocatalyst surface and the free catalytic center $\left(\left[\mathrm{Cp} * \mathrm{Rh}(\mathrm{bpy}) \mathrm{H}_{2} \mathrm{O}\right]^{2+}\right.$, denoted as Rh). Meanwhile, the presence of free catalytic center further increases subsequent difficulty and cost for target product recovery. Therefore, it is imperative to engineer the photocatalyst with enhanced electron utilization efficiency for nicotinamide cofactor regeneration.

In nature photosynthesis, ferredoxin- $\mathrm{NADP}^{+}$reductase (FNR), as part of thylakoid membrane anchoring on the surface (Figure 1(a)), is the electron utilization center during the regeneration of reduced nicotinamide adenine dinucleotide phosphate (NADPH) [20]. Under visible light irradiation, electrons are generated and transferred to protein complex photosystem I (PS I) through an electron transfer chain [11] and stored in ferredoxin (Fd), which is directly connected with three subunits of PS I [21]. Then, the electrons are transferred from a $2 \mathrm{Fe}-2 \mathrm{~S}$ cluster of $\mathrm{Fd}$ to flavin adenine dinucleotide (FAD) prosthetic group of FNR, the ultimate destination in electron transfer chain, for utilization in the regeneration of NADPH. In particular, the distance between $2 \mathrm{Fe}-2 \mathrm{~S}$ cluster and FAD is extremely short, which relies on the electrostatic interactions formed through salt bridges. The electron transfer distance from photosensitizer to the electron utilization center is shortened for nanoconfinement between Fd and FNR, which elevates the electron utilization. The unique mode of anchored FNR guarantees about $100 \%$ of the inherent quantum efficiency for natural photosynthesis [22]. 
Inspired by the structure and function of FNR anchored on thylakoid membrane, a tannic acid/polyethyleneimine (TA/PEI) nanoshell anchored with $\left[\mathrm{Cp} * \mathrm{Rh}(\mathrm{bpy}) \mathrm{H}_{2} \mathrm{O}\right]^{2+}$ $(\mathrm{Rh})$ is formed on the surface of polymeric carbon nitride (PCN) through polyphenol-induced surface adhesion process [23-25]. The acquired core@shell photocatalyst was denoted as PCN@TA/PEI-Rh (Figure 1(b)). The PCN, a typical metal-free semiconductor material [26], is chosen as photoresponsive core to generate electrons under visible light illumination, similar to PS I on thylakoid membrane. Then, the photoexcited electrons are transferred and utilized by the Rh immobilized on TA/PEI nanoshell for nicotinamide cofactor regeneration, similar to FNR anchored on thylakoid membrane. The surface-anchored Rh intensifies utilization of photoexcited electrons arisen from the shortened electron transfer distance between Rh and PCN, which can also simplify nicotinamide cofactor regeneration system. The content of immobilized Rh can be facilely controlled by altering the chemical compositions of TA/PEI nanoshell. The photoenzymatic conversion of carbon dioxide $\left(\mathrm{CO}_{2}\right)$ could be realized through coupling PCN@TA/PEI-Rh with formate dehydrogenase $(\mathrm{FDH})$. Combined with the controllability and mild condition of polyphenol-induced adhesion method, our study may offer a generic strategy to improve the photocatalytic efficiency via intensifying the electron utilization.

\section{Results}

2.1. Preparation and Characterizations of PCN@TA/PEI-Rh Core@Shell Photocatalyst. In our study, the PCN@TA/PEIRh core@shell photocatalyst was prepared through anchoring an $\mathrm{Rh}$ complex $\left(\left[\mathrm{Cp} * \mathrm{Rh}(\text { bpy }) \mathrm{H}_{2} \mathrm{O}\right]^{2+}\right.$, a molecular catalyst for selective hydrogenation of pyridine ring) on polymeric carbon nitride (PCN) mediated by a polyphenolinspired adhesive layer [24]. The general preparation process is demonstrated in Figure 1(c). Briefly, TA, a typical polyphenol, was adhered on the PCN surface and further crosslinked with PEI triggered by a pH change, forming the PCN@TA/ PEI particle. Then, the coupling reaction between PEI moiety in the TA/PEI nanoshell and bipyridine carboxylate (bpy$\mathrm{COOH}$ ) occurred with the addition of N-hydroxysuccinimide (NHS) and 1-(3-dimethylaminopropyl)-3-ethylcarbodiimide hydrochloride (EDC), where PCN@TA/PEI-bpy particle was obtained. Finally, PCN@TA/PEI-Rh core@shell photocatalyst was obtained via the coordination of bipyridine and $\left(\mathrm{Cp} * \mathrm{RhCl}_{2}\right)_{2}$ (Figure S1). As shown in Figure S2a, PCN@TA/PEI-Rh exhibited a typical bulky structure, where $\mathrm{C}, \mathrm{N}, \mathrm{O}$, and $\mathrm{Rh}$ elements were uniformly distributed (Figure S2b-e). Particularly, the presence of $\mathrm{O}$ and Rh element preliminary validated the existence of TA molecules and Rh complex on the surface of PCN core. In order to visually observe the thickness of TA/PEI nanoshell, $\mathrm{SiO}_{2}$ core with a regular sphere structure was used instead of PCN to prepare $\mathrm{SiO}_{2} @ \mathrm{TA} / \mathrm{PEI}$. Clearly, the TA/PEI nanoshell with a thickness of $\sim 10 \mathrm{~nm}$ was observed in the TEM image (Figure 1(c)).

Subsequently, the chemical and physical compositions of PCN@TA/PEI-Rh were examined by Fourier transform infrared spectroscopy (FTIR), X-ray photoelectron spectros- copy (XPS), and X-ray powder diffraction (XRD). Commonly, PCN prepared by thermal polycondensation of melamine showed a graphite-like lamellar structure, of which the PCN layers had a triazine ring structure and co-ssembled via hydrogen bonds and $\pi-\pi$ interaction. As shown in Figure S3, PCN exhibited two strong peaks at $12.9^{\circ}$ and $27.4^{\circ}$ in the XRD pattern, which were assigned to the $\pi-\pi$ conjugated structures and tri-s-triazine structures of PCN, respectively. The existence of triazine ring in PCN was also validated by its absorption band at $806 \mathrm{~cm}^{-1}$ in the FTIR spectrum (Figure S4). Moreover, the absorption bands at $3000-3500 \mathrm{~cm}^{-1}$ and $1234-1635 \mathrm{~cm}^{-1}$ were, respectively, corresponded to $\mathrm{N}-\mathrm{H}$ stretching and $\mathrm{C}-\mathrm{N} / \mathrm{C}=\mathrm{N}$ stretching, further indicating the successful preparation of PCN. As for PCN@TA/PEI and PCN@TA/PEI-Rh, the absorption bands that should be assigned to $\mathrm{Ar}-\mathrm{OH}\left(1200 \mathrm{~cm}^{-1}\right)$ and $\mathrm{C}=\mathrm{O}$ $\left(1717 \mathrm{~cm}^{-1}\right)$ in the TA molecules were not observed due to the interference of the absorption bands of PCN. Fortunately, the presence of $\mathrm{O}$ 1s peak in the XPS survey spectra of PCN@TA/PEI and PCN@TA/PEI-Rh (Figure 2(a)) verified the existence of TA molecules on PCN. The anchoring of $\mathrm{Rh}$ on PCN was also validated by the presence of Rh $3 \mathrm{~d}$ peak in the XPS survey spectra of PCN@TA/PEI-Rh (Figure 2(b)). Specifically, the peaks appeared at $314.0 \mathrm{eV}$ and $309.3 \mathrm{eV}$ were assigned to the binding energies of $\mathrm{Rh} 3 \mathrm{~d}_{2 / 3}$ and $\mathrm{Rh} 3 \mathrm{~d}_{5 / 2}$, respectively (Figure 2(b)). High-resolution C 1s XPS spectra of PCN@TA/PEI-Rh (Figure S5) could be deconvoluted to four peaks at $287.9 \mathrm{eV}, 286.2 \mathrm{eV}, 285.3 \mathrm{eV}$, and $284.5 \mathrm{eV}$. The main peaks at $287.9 \mathrm{eV}$ and $284.5 \mathrm{eV}$ were assigned to the $\mathrm{N}-\mathrm{C}=\mathrm{N}$ bond and $\mathrm{C}-\mathrm{C}$ bond of $\mathrm{PCN}$, respectively. Two new peaks at 286.2 and $285.3 \mathrm{eV}$ were attributed to the $\mathrm{C}$ atom of $\mathrm{C}-\mathrm{O}$ bond and $\mathrm{C}-\mathrm{N}$ bond of TA and PEI, respectively. For $\mathrm{O}$ 1s spectra (Figure S6), two new peaks of PCN@TA/PEI-Rh at $532.2 \mathrm{eV}$ and $530.8 \mathrm{eV}$ were clearly observed, corresponding to $\mathrm{O}$ of $\mathrm{C}=\mathrm{O}$ bond and $\mathrm{C}-\mathrm{O}$ bond in TA, respectively. The $\mathrm{N}$ 1s spectra of PCN, PCN@TA/PEI, and PCN@TA/PEI-Rh (Figure 2(c)) all showed the characteristic peaks of $\mathrm{C}-\mathrm{NH}_{2}, \mathrm{~N}-(\mathrm{C})_{3}$, and $\mathrm{C}-\mathrm{N}=\mathrm{C}$. In particular, the characteristic peaks at $400.8 \mathrm{eV}$, $399.3 \mathrm{eV}$, and $398.3 \mathrm{eV}$ of PCN@TA/PEI-Rh slightly shifted compared to the characteristic peaks at $401.3 \mathrm{eV}, 399.6 \mathrm{eV}$, and $398.8 \mathrm{eV}$ of PCN@TA/PEI, which revealed the existence of chemical interaction between $\mathrm{Rh}$ and TA/PEI nanoshell.

The band structures of PCN, PCN@TA/PEI, and PCN@TA/PEI-Rh were then characterized by XPS valence band (VB) spectra, the Mott-Schottky plot, and UV-vis diffuse reflection spectroscopy (DRS). As shown in Figure S7, the absorption edge of PCN@TA/PEI-Rh (482 nm) was wider than that of PCN $(456 \mathrm{~nm})$ and PCN@TA/PEI $(464 \mathrm{~nm})$. The PCN@TA/PEI-Rh exerted narrower band gap of $2.67 \mathrm{eV}$ in the UV-vis DRS spectra (Figure 2(d)), indicating that the TA/PEI nanoshell grafted with $\mathrm{Rh}$ widened the visible light absorption range of PCN. The conduction band (CB) values of PCN, PCN@TA/PEI, and PCN@TA/PEI-Rh detected by Mott-Schottky plots (Figure 2(e)) were $-1.195 \mathrm{~V},-1.164 \mathrm{~V}$, and $-1.141 \mathrm{~V}$ vs. $\mathrm{Ag} / \mathrm{AgCl}, \mathrm{pH}=7.0$, respectively (equal to $-1.195 \mathrm{~V},-1.164 \mathrm{~V}$, 


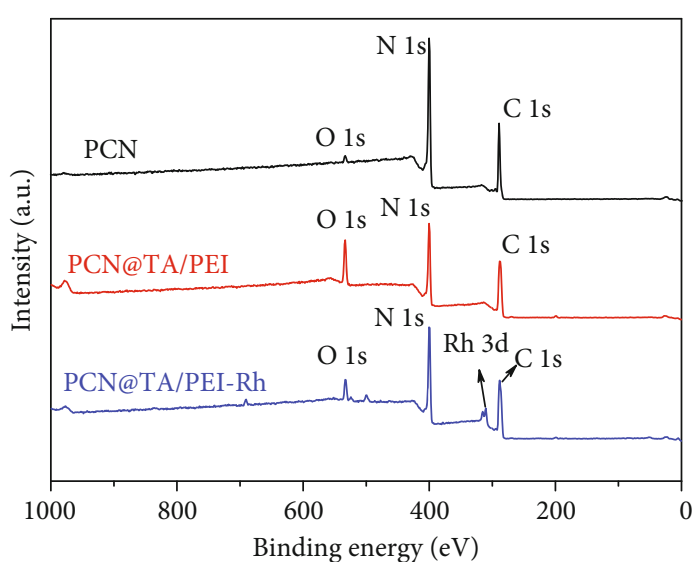

(a)

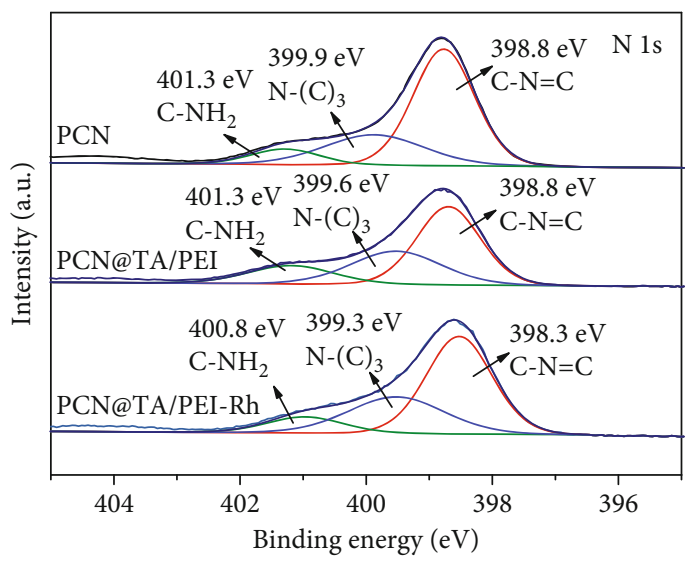

(c)

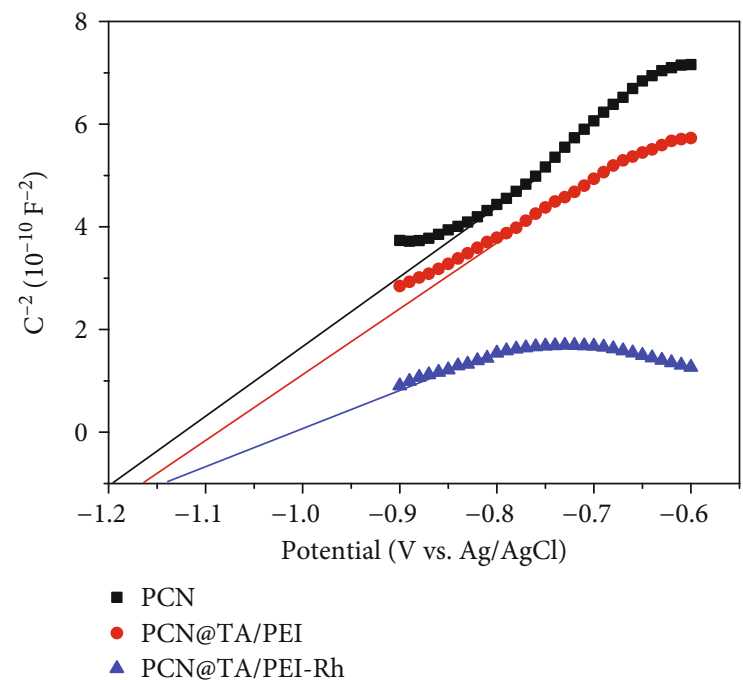

(e)

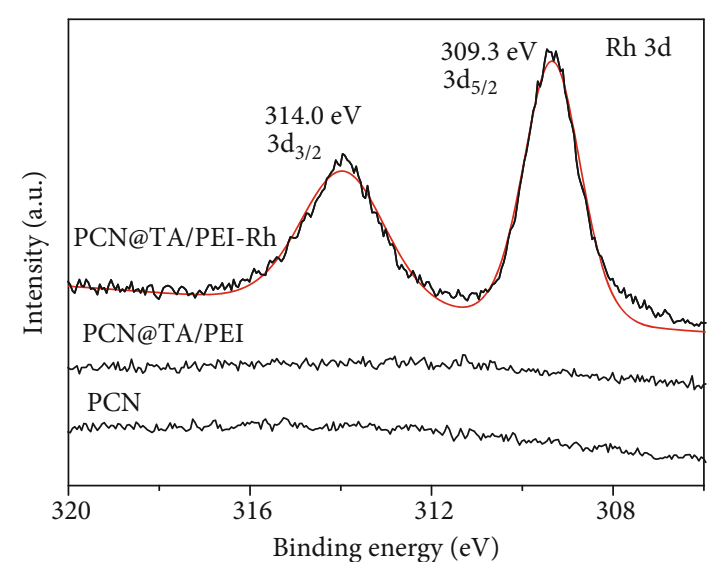

(b)

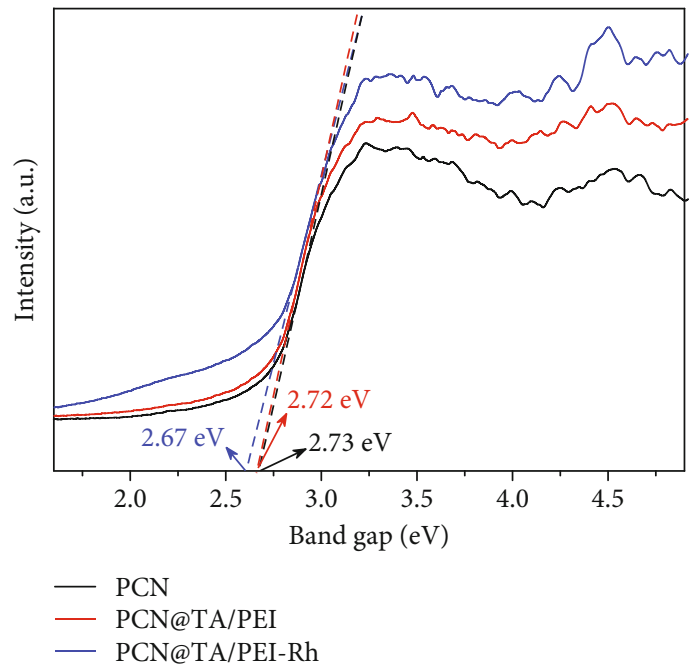

(d)

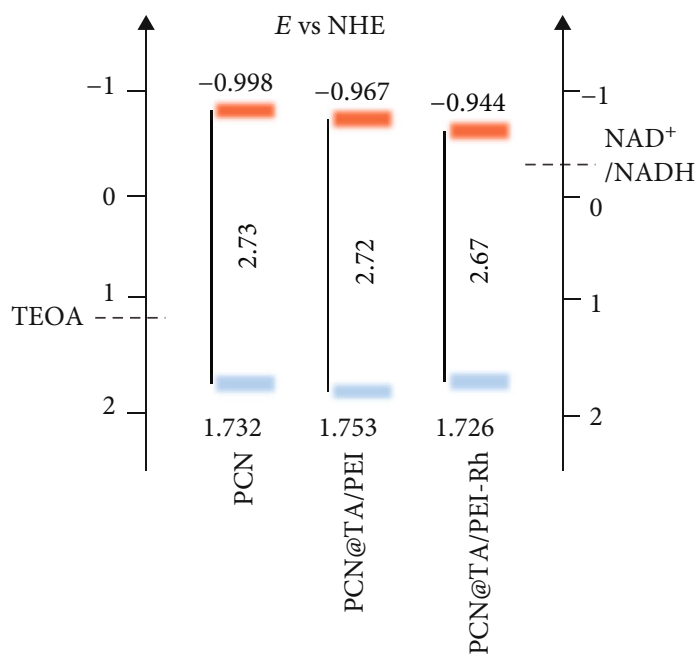

(f)

Figure 2: (a) XPS survey spectra of PCN, PCN@TA/PEI, and PCN@TA/PEI-Rh. High-resolution XPS (b) Rh 3d and (c) N 1s spectra of PCN, PCN@TA/PEI, and PCN@TA/PEI-Rh. (d) Calculated band gap patterns based on UV-vis diffuse reflectance spectra. (e) Mott-Schottky plots of PCN, PCN@TA/PEI, and PCN@TA/PEI-Rh. (f) Conduction (orange) and valence (blue) band-edge positions of PCN, PCN@TA/PEI, and PCN@TA/PEI-Rh. 
and-1.141 V vs. NHE, $\mathrm{pH}=7.0$ ). According to band gap and CB analysis, the VB potentials of PCN, PCN@TA/PEI, and PCN@TA/PEI-Rh were calculated to $1.732 \mathrm{~V}, 1.753 \mathrm{~V}$, and $1.726 \mathrm{~V}$ (vs. NHE, $\mathrm{pH}=7.0$ ), respectively. It should be noted that the redox potential of $\mathrm{NAD}^{+} / \mathrm{NADH}$ was $-0.32 \mathrm{~V}$ (vs. NHE, $\mathrm{pH}=7.0$ ) [11], which was lower than the CB of PCN, PCN@TA/PEI, and PCN@TA/PEI-Rh (Figure 2(f)). This indicated that PCN, PCN@TA/PEI, and PCN@TA/PEI-Rh could be able to regenerate $\mathrm{NADH}$.

The transfer and utilization processes of photoexcited electrons of PCN and PCN@TA/PEI-Rh were elucidated by photoelectrochemical analysis. As shown in Figure S8, PCN@TA/PEI-Rh excited by light showed 150\% elevation of the carrier density by contrast with PCN. This suggested the transfer of more photoexcited electrons from bulk to surface. More electrons could then be readily used. As for the PL spectra, the PCN@TA/PEI-Rh displayed a much lower absorption peak at $470 \mathrm{~nm}$ compared with PCN (Figure S9), indicating a lower electron-hole recombination. We could then conjecture that more photoexcited electrons were captured by the anchored Rh, which was beneficial for improving the utilization efficiency of transferred electrons. Hence, we further calculated the Hirshfeld charge of PCN@TA/PEI and PCN@TA/PEI-Rh through DFT. As shown in Figure S10, to simplify the model, bpy-H and bpy-Rh-H were used to represent PCN@TA/PEI and PCN@TA/PEI-Rh, respectively, for examining the role of Rh. Accordingly, two models of bpy-H and bpy-Rh-H were optimized by Gaussian 03 program. Calculated with Multiwfn, the Hirshfeld charge of the additional $\mathrm{H}$ atom in bpy- $\mathrm{Rh}-\mathrm{H}$ was -0.06 while that in bpy- $\mathrm{H}$ is more positive (0.15). The calculation indicated that more electrons were enriched on the surface-anchored $\mathrm{Rh}$, so that more electrons could be utilized for NADH regeneration.

To figure out whether the TA/PEI moiety affects the electron transfer or utilization behavior, the electron transfer behaviors of PCN, PCN@TA/PEI, and PCN@TA/PEI-Rh were monitored by EIS Nyquist plots and time-resolved transient PL spectra. As shown in Figure 3(a), the curve radius of PCN@TA/PEI-Rh was less than PCN and PCN@TA/PEI. That illustrated the lower interfacial electron transfer resistance of PCN@TA/PEI-Rh, which could promote electron transfer rate for PCN@TA/PEI-Rh. In the time-resolved transient PL spectra (Figure 3(b)), the decay curves of PCN, PCN@TA/PEI, and PCN@TA/PEI-Rh were fitted by the following biexponential equation:

$$
\mathrm{I}(t)=A+B_{1} \exp \left(-\frac{\mathrm{t}}{\tau_{1}}\right)+B_{2} \exp \left(-\frac{t}{\tau_{2}}\right)
$$

where $B_{1}$ and $B_{2}$ were the corresponding amplitudes and $\tau_{1}$ and $\tau_{2}$ were the fluorescent lifetimes. The average fluorescent lifetime was described in the following equation:

$$
\tau=\frac{\mathrm{B}_{1} \tau_{1}^{2}+B_{2} \tau_{2}^{2}}{B_{1} \tau_{1}+B_{1} \tau_{2}}
$$

With parameters in Table S1, the fluorescence lifetime of PCN, PCN@TA/PEI, and PCN@TA/PEI-Rh could be calculated to $0.906 \mathrm{~ns}, 0.847 \mathrm{~ns}$, and $0.743 \mathrm{~ns}$, respectively, further evidencing the electron transfer from PCN to Rh through TA/PEI nanoshell. All above results evidenced that the TA/PEI moiety did not alter the electron transfer or utilization process, whereas the anchoring of Rh captured more electrons for further utilization. We then quantitatively analyzed the electron utilization efficiency (EUE) by the methods as reported in our previous work [15]. As shown in Figure 3(c), the open-circuit potential (OCP) of PCN and PCN@TA/PEI-Rh was measured. During the illumination time of $100 \mathrm{~s}$, the voltage increased with the accumulation of holes. After irradiation, the reduced voltage indicated the recombination of electrons and holes. The EUE of PCN@TA/PEI-Rh was calculated to $61.4 \%$ with the following equation3, achieved $\sim 1.3$-fold elevation compared with that of PCN (48.6\%).

$$
\mathrm{EUE}=\left(\frac{V_{2}-V_{0}}{V_{1}-V_{0}}\right) \times 100 \%
$$

where $V_{0}, V_{1}$, and $V_{2}$ are the OCPs at 100,200 , and $600 \mathrm{~s}$, respectively. In the cyclic voltammetry curve of PCN and PCN@TA/PEI-Rh (Figure 3(d)), a wider closed curve of PCN@TA/PEI-Rh was detected and a redox peak at $-0.849 \mathrm{~V}$ was observed (vs. $\mathrm{Ag} / \mathrm{AgCl}$ ), indicating that the redox reaction occurred when electrons were transferred from PCN to Rh.

2.2. Nicotinamide Cofactor Regeneration and Photoenzymatic $\mathrm{CO}_{2}$ Conversion. During the photoenzymatic process, NADH (reduced nicotinamide adenine dinucleotide, a typical nicotinamide cofactor) is frequently used as the "energy currency" to bridge photocatalysis and enzyme catalysis [27]. The efficient regeneration of NADH by photocatalyst is thus urgently required. As shown in Figure 4(a), the $\left[\mathrm{Cp} * \mathrm{Rh}(\mathrm{bpy}) \mathrm{H}_{2} \mathrm{O}\right]^{2+}(\mathrm{Rh})$ was used as electron mediator and triethanolamine (TEOA) was used as electron donor to perform photocatalytic NADH regeneration. Under visible light illumination, the photoexcited electrons on PCN were transferred to $\mathrm{Rh}$ on the TA/PEI nanoshell and then triggered the regeneration of $\mathrm{NADH}$, whereas the holes on PCN were quenched by TEOA to suppress the electronhole recombination. The efficiency of regenerated $\mathrm{NADH}$ was calculated by detecting the absorbance of $\mathrm{NADH}$ at $340 \mathrm{~nm}$ with UV-visible spectroscopy and verified by enzyme assay.

In our study, the chemical structures of nanoshell were firstly optimized through changing the ratios of TA concentration to $\mathrm{PEI}$ concentration $([\mathrm{TA}] /[\mathrm{PEI}])$ and coupling reaction time $(X)$ of PEI and bpy-COOH to investigate their effect on NADH regeneration efficiency. Specifically, three samples of PCN@TA/PEI-Rh with different [TA]/[PEI] were prepared. As shown in Figure 4(b), the optimized NADH regeneration efficiencies of three PCN@TA/PEI-Rh were all presented at $20 \mathrm{~min}$. With the increase of [TA]/[PEI] from $1 / 1$ to $1 / 10$, the optimized $\mathrm{NADH}$ regeneration efficiency 


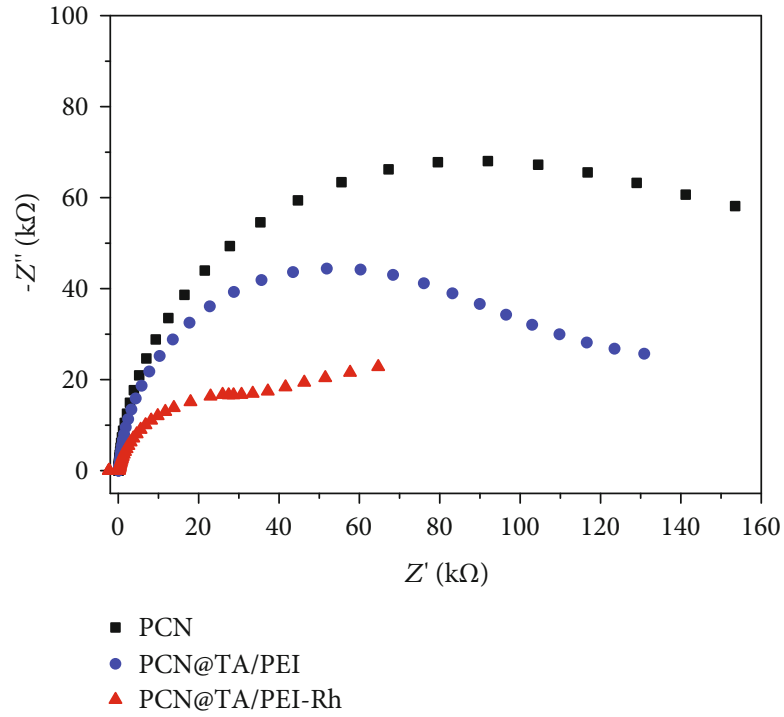

(a)

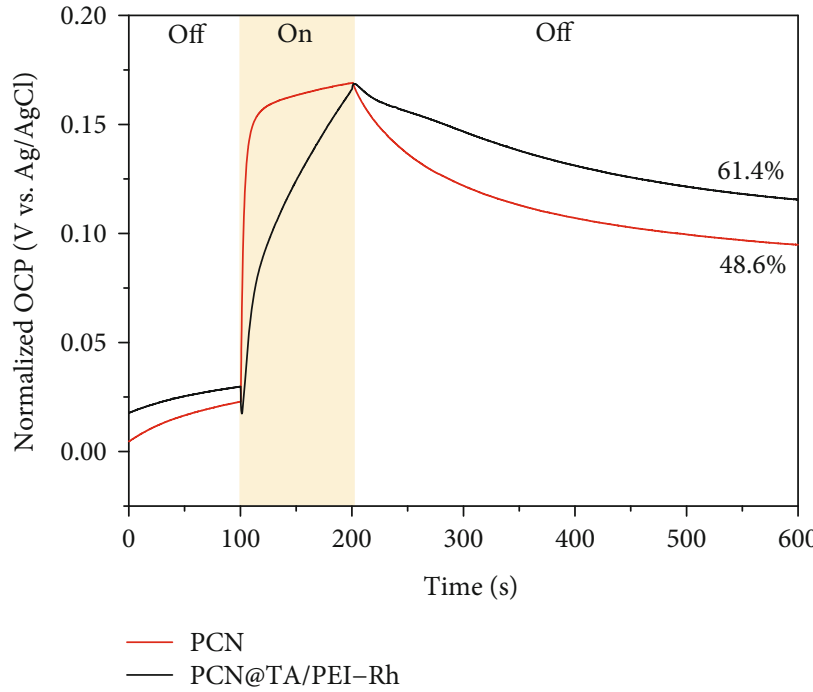

(c)

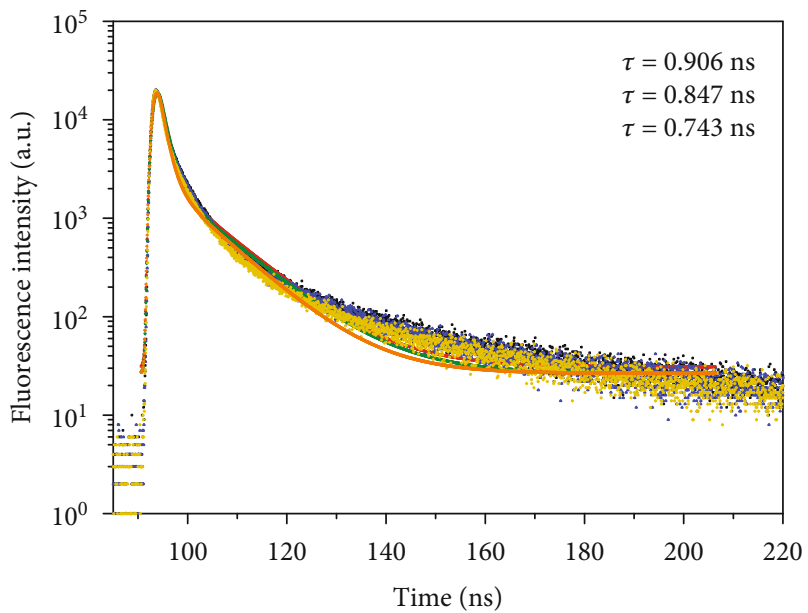

- PCN

- PCN@TA/PEI

- PCN@TA/PEI-Rh

(b)

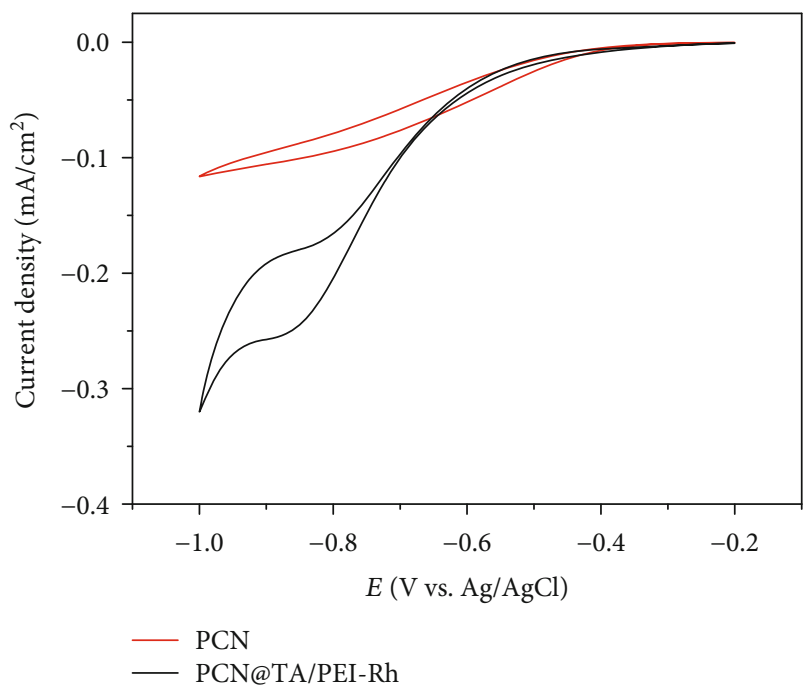

(d)

Figure 3: (a) EIS Nyquist plots of PCN, PCN@TA/PEI, and PCN@TA/PEI-Rh in $0.1 \mathrm{M} \mathrm{Na}_{2} \mathrm{SO}_{4}$ without illumination. (b) Time-resolved transient PL spectra of PCN, PCN@TA/PEI, and PCN@TA/PEI-Rh. (c) Open-circuit potential of PCN and PCN@TA/PEI-Rh as a function of time. (d) Cyclic voltammetry curve of PCN and PCN@TA/PEI-Rh at a scan rate of $5 \mathrm{mV} \mathrm{s}^{-1}$.

increased from $7.8 \%$ to $37.8 \%$. This was probably due to the increase of $\mathrm{Rh}$ content from $0.020 \mathrm{mM}$ to $0.028 \mathrm{mM}$ as detected by inductively coupled plasma spectrometer (ICPOES, Figure S11). Subsequently, the NADH regeneration efficiency further slightly decreased from $37.8 \%$ to $29.8 \%$ with the increase of $[\mathrm{TA}] /[\mathrm{PEI}]$ from $1 / 10$ to $1 / 20$ as evidenced by the slight decrease of $\mathrm{Rh}$ content from $0.028 \mathrm{mM}$ to $0.027 \mathrm{mM}$ (Figure S11). Then, the photocatalytic performance of PCN@TA/PEI-Rh was optimized by altering the $X$ of PEI and bpy-COOH. The extension of $X$ was beneficial to the efficiency of photocatalytic NADH regeneration (Figure S12). Specially, the NADH regeneration efficiency unaltered when $X$ was over $48 \mathrm{~h}$, indicating that the coupling reaction between amino and carboxyl groups was completed at $48 \mathrm{~h}$.
Based on above analysis, the NADH regeneration efficiencies of different photocatalysts with free $\mathrm{Rh}$ (the content of free Rh was the same as that of PCN@TA/PEI-Rh $\mathrm{R}_{1 / 10}$ ) and surface-anchored $\mathrm{Rh}$ were measured. The reaction setup is shown in Figure S13. As shown in Figure 4(b), the optimized NADH regeneration efficiency of PCN@TA/PEI$\mathrm{Rh}(37.8 \%)$ was $156 \%$ higher than that of PCN with free Rh (24.2\%), which should be arisen by the higher EUE. Meanwhile, the turnover frequency (TOF) of PCN@TA/ PEI-Rh ${ }_{1 / 1}$, PCN@TA/PEI-Rh ${ }_{1 / 1}$, PCN@TA/PEI-Rh ${ }_{1 / 20}$, PCN, and PCN@TA/PEI with free Rh system was calculated as shown in Figure 4(c). By contrast, the PCN@TA/PEI-Rh $\mathrm{R}_{1 / 10}$ showed TOF of $70.82 \mathrm{~h}^{-1}$ based on Rh, which is, to the best of our knowledge, the highest rate among the reported photocatalytic NADH regeneration (Table S2). 


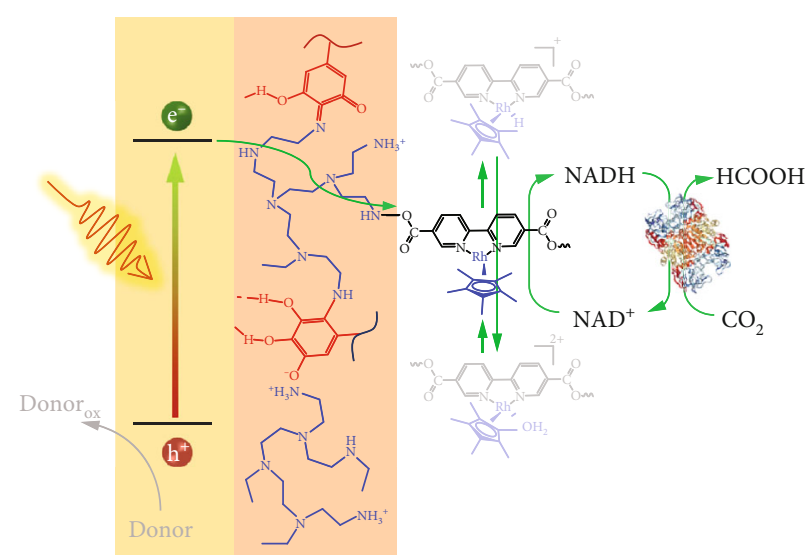

(a)

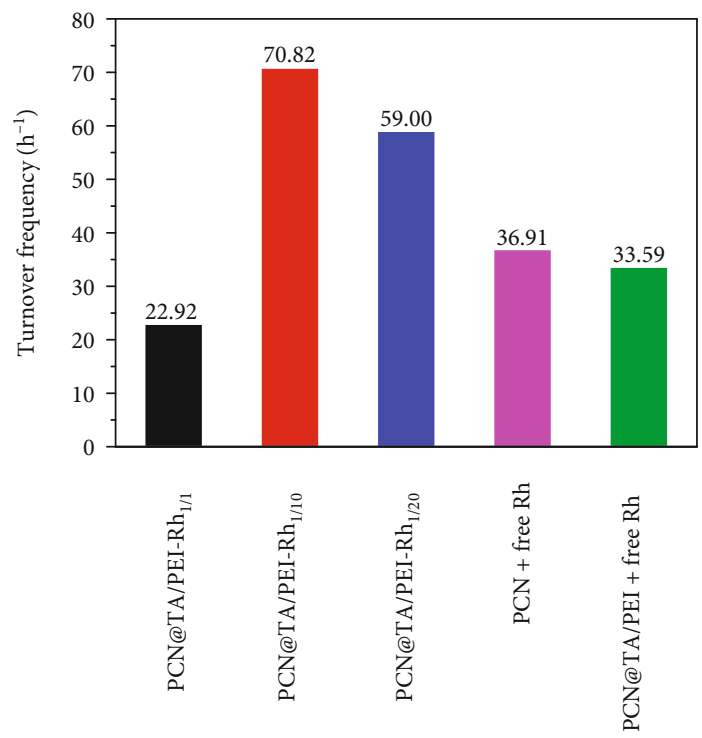

(c)

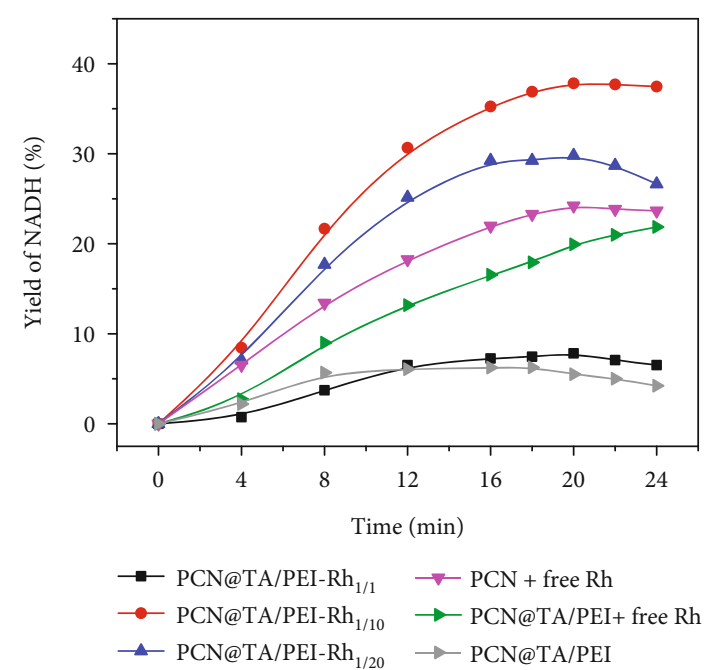

(b)

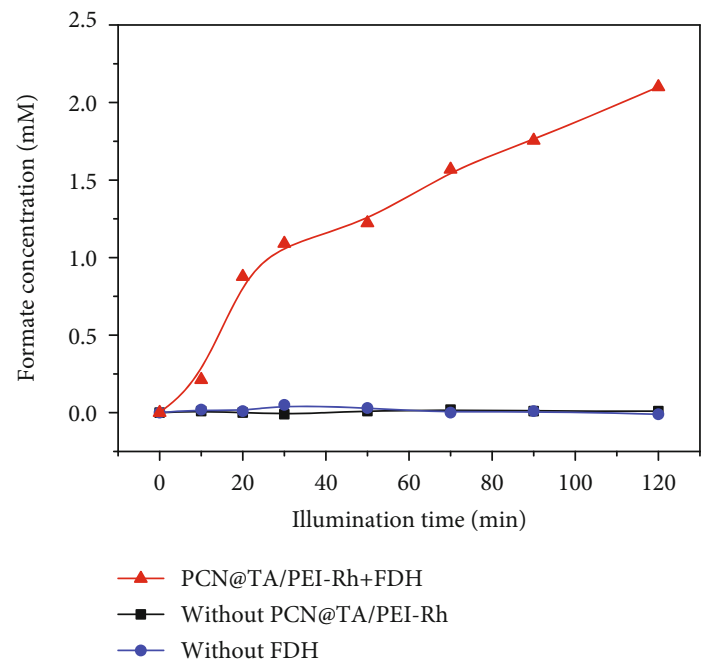

(d)

FIGURE 4: (a) Scheme of nicotinamide cofactor regeneration system. (b) The efficiency of NADH catalyzed by PCN@TA/PEI-Rh different $[\mathrm{TA}] /[\mathrm{PEI}], \mathrm{PCN}$, and PCN@TA/PEI with free Rh system. (c) The turnover frequency (TOF) of PCN@TA/PEI-Rh ${ }_{1 / 1}$, PCN@TA/PEI-Rh $1 / 10$, PCN@TA/PEI-Rh ${ }_{1 / 20}$, PCN, and PCN@TA/PEI with free Rh system. (d) Photoenzymatic conversion of CO 2 enabled by PCN@TA/PEI-Rh. Reaction conditions: (b) [photocatalyst] $=0.5 \mathrm{mg} \mathrm{mL}{ }^{-1}, \mathrm{PBS}$ buffer $(\mathrm{pH} 7.0,100 \mathrm{mM}),\left[\mathrm{NAD}{ }^{+}\right]=1 \mathrm{mM},[$ free $\mathrm{Rh}]=0.028 \mathrm{mM},[\mathrm{TEOA}]=400 \mathrm{mM}$, visible light illumination $(\lambda=405 \mathrm{~nm})$, volume $=2 \mathrm{~mL}$, and room temperature; $(\mathrm{d})[\mathrm{PCN} @ \mathrm{TA} / \mathrm{PEI}-$ $\mathrm{Rh}]=0.5 \mathrm{mg} \mathrm{mL}^{-1},[\mathrm{FDH}]=2 \mathrm{mg} \mathrm{mL}^{-1},\left[\mathrm{NAD}^{+}\right]=5 \mathrm{mM}$, and $\left[\mathrm{CO}_{2}\right]=0.3 \mathrm{MPa}$.

To further in-depth understand the NADH regeneration process, the reaction kinetics were analyzed based on the experiment measured at different $\mathrm{NAD}^{+}$concentrations (0.1 mM-0.5 mM) (Table S3) [28]. In general, the photocatalytic NADH regeneration process with PCN@TA/ PEI-Rh could be categorized into three primary steps (Figure 5): (1) redox reaction between TEOA and catalyst (donated as $\mathrm{C} *$, the catalyst in its oxidized form); (2) redox reaction between catalyst and $\mathrm{Rh}$; and (3) redox reaction between $\mathrm{Rh}-\mathrm{H}$ (the $\mathrm{Rh}$ in its hydride form) and $\mathrm{NAD}^{+}$. Given the above conditions, the mathematical equations relating $r_{\mathrm{NADH}}(d[\mathrm{NADH}] / d t)$ to $\left[\mathrm{NAD}^{+}\right]$are shown in the following equations, imposing the steady-state concentration to $\mathrm{C}$ and $\mathrm{Rh}$ (as detailed in Supplementary Materials).

$$
\begin{aligned}
& r_{\mathrm{NADH}}= \frac{d[\mathrm{NADH}]}{d t}=k_{3}[\mathrm{Rh}-\mathrm{H}]\left[\mathrm{NAD}^{+}\right], \\
& k_{1} k_{3}[\mathrm{TEOA}]\left[\mathrm{NAD}^{+}\right][\mathrm{Rh}-\mathrm{H}] \\
&=\left(\mathrm{C}_{\mathrm{Rh}}-[\mathrm{Rh}-\mathrm{H}]\right)\left(k_{1} k_{2} \mathrm{C}_{\mathrm{C}}[\mathrm{TEOA}]\right. \\
& \\
&\left.-k_{2} k_{3}\left[\mathrm{NAD}^{+}\right][\mathrm{Rh}-\mathrm{H}]\right) .
\end{aligned}
$$




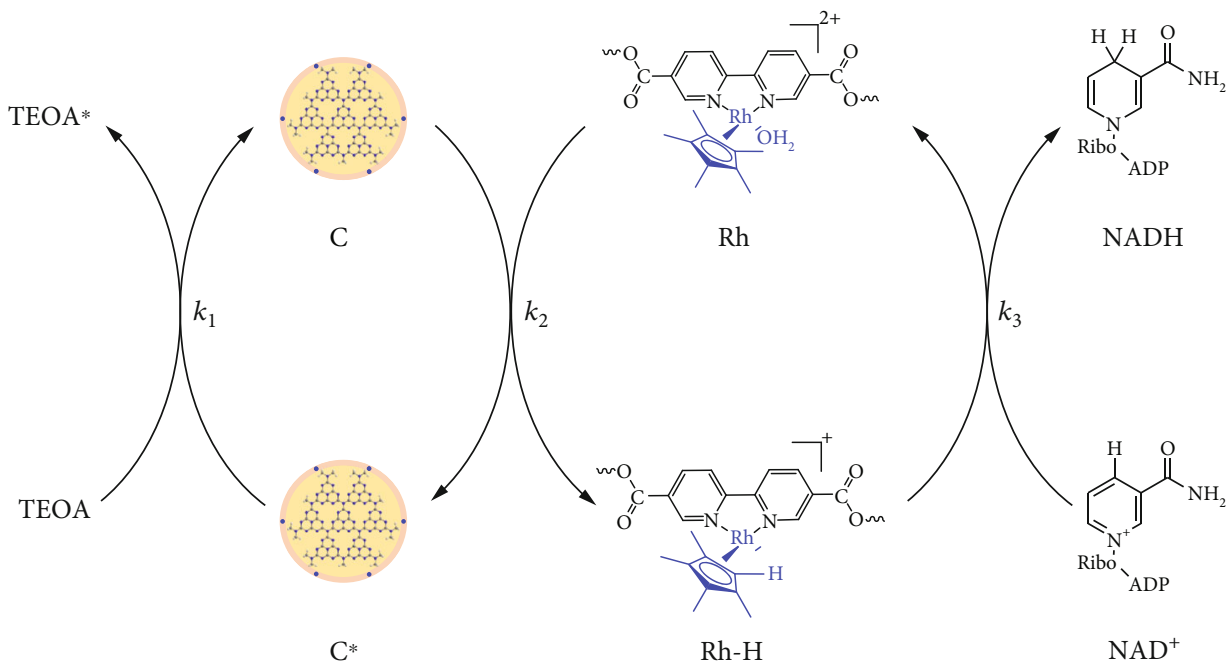

FIGURE 5: Reaction mechanism of photocatalytic NADH regeneration with PCN@TA/PEI-Rh.

TABLE 1: Kinetic constants calculated by Equations (4) and (5) with the experimental data of PCN and PCN@TA/PEI-Rh.

\begin{tabular}{lcc}
\hline Kinetic constants & PCN+free Rh & PCN@TA/PEI-Rh \\
\hline$k_{1}\left(\mathrm{M}^{-1} \mathrm{~min}^{-1}\right)$ & $3.68 \times 10^{-5}$ & $3.32 \times 10^{-4}$ \\
$k_{2}\left(\mathrm{M}^{-1} \mathrm{~min}^{-1}\right)$ & 0.57 & 12.45 \\
$k_{3}\left(\mathrm{M}^{-1} \mathrm{~min}^{-1}\right)$ & 750.00 & 213.08 \\
\hline
\end{tabular}

Substituting the experimental data $r_{\mathrm{NADH}}$ versus [NAD ${ }^{+}$] into Equations (4) and (5), $k_{1}, k_{2}$, and $k_{3}$ were obtained (Table 1). The result confirmed the superiority of PCN@TA/ PEI-Rh as evidenced by the higher capability of surfaceanchored Rh to utilize electrons $\left(k_{2}\right.$ of PCN@TA/PEI-Rh was 20 times larger than that for PCN) and lower density of holes to inhibit recombination $\left(k_{1}\right.$ of PCN@TA/PEI-Rh was 10 times larger than that of $\mathrm{PCN})$. As for the reaction between Rh-H and $\mathrm{NAD}^{+}\left(k_{3}\right.$ in Table 1$), \mathrm{PCN} @ \mathrm{TA} / \mathrm{PEI}-\mathrm{Rh}$ was inactive than $\mathrm{PCN}$ arising from the transition from liquidliquid homogeneous phase to solid-liquid heterogeneous phase between $\mathrm{Rh}$ and $\mathrm{NAD}^{+}$, but the difference is less critical ( $k_{3}$ was 3.5 times smaller than for $\mathrm{PCN}$ ).

Finally, the recycling stability of PCN@TA/PEI-Rh was investigated. After 5 cycles, $\sim 52 \%$ activity of $\mathrm{NADH}$ regeneration efficiency was retained (Figure S14). To assess the enzymatic activity of regenerated $\mathrm{NADH}$, formate dehydrogenase (FDH) was coupled with PCN@TA/PEI-Rh for photoenzymatic conversion of $\mathrm{CO}_{2}$ into formate [29]. As shown in Figure 4(d), the PCN@TA/PEI-Rh and FDHcoupled system produced $2.1 \mathrm{mM}$ formate in $120 \mathrm{~min}$, and each NADH was used 2.32 times for formate production (calculated with Equation (8)). By contrast, in the absence of PCN@TA/PEI-Rh or FDH, no accumulation of formate was detected.

\section{Discussion}

In summary, the PCN@TA/PEI-Rh core@shell photocatalyst was prepared by anchoring Rh complex on the PCN core through polyphenol-induced adhesion method for lightdriven NADH regeneration and photoenzymatic $\mathrm{CO}_{2}$ conversion. Under visible light illumination, the PCN core was excited to generate electrons, whereas the surface-anchored $\mathrm{Rh}$ on TA/PEI nanoshell acted as the electron utilization center toward NADH regeneration. The electron utilization was remarkably fortified by the surface-anchored $\mathrm{Rh}$ as validated by open-circuit potential, which could be regulated by changing the $[\mathrm{TA}] /[\mathrm{PEI}]$ ratio and coupling reaction time of PEI and bpy-COOH. Under optimal condition, the PCN@TA/ PEI-Rh exerted a 1.3-fold elevation in EUE compared with the system where $\mathrm{Rh}$ was in free form, leading to a photocatalytic NADH regeneration efficiency of $\sim 37.8 \%$. Photoenzymatic conversion of $\mathrm{CO}_{2}$ could then be realized by coupling PCN@TA/PEI-Rh with formate dehydrogenase (FDH). Hopefully, our study may provide a generic strategy to intensify electron utilization for enhanced photocatalytic performance.

\section{Materials and Methods}

4.1. Materials. Pentamethylcyclopentadienyl rhodium (III) chloride dimer $\left(\left(\mathrm{Cp} * \mathrm{RhCl}_{2}\right)_{2}\right)$ and triethanolamine (TEOA) were purchased from Aladdin Industrial Corporation (Shanghai, China). Tannic acid (TA), N-hydroxysuccinimide (NHS), and 2,2' -bipyridine-5, $5^{\prime}$-dicarboxylic acid were purchased from J\&K Chemical (Shanghai, China). $\beta$-Nicotinamide adenine dinucleotide phosphate sodium salt hydrate $\left(\mathrm{NAD}^{+}\right)$, formate dehydrogenase (FDH) from Candida boidinii, melamine, polyethyleneimine (PEI; average $\mathrm{Mw}$ $\sim 2000 \mathrm{Da}$ ), tris(hydroxymethyl)aminomethane (Tris), and silica $\left(\mathrm{SiO}_{2}\right.$ powder, $\left.0.2-0.3 \mu \mathrm{m}\right)$ were purchased from Sigma-Aldrich (St. Louis, USA). 1-(3-Dimethylaminopropyl)-3-ethylcarbodiimide hydrochloride (EDC) was purchased from TCI Chemical (Shanghai, China). All other materials were used without further purification.

4.2. Preparation of PCN. Polymeric carbon nitride (denoted as PCN) was synthesized by the condensation reaction of 
melamine with a one-step heated treatment. Firstly, melamine was weighed in a small crucible, which was sealed and wrapped with three layers of tin foil. Then, the crucible was stayed in a muffle furnace at $550^{\circ} \mathrm{C}$ for $4 \mathrm{~h}$, of which the temperature was increased to $550^{\circ} \mathrm{C}$ at a rate of $5^{\circ} \mathrm{C} \mathrm{min}{ }^{-1}$. Finally, the yellow PCN was obtained after the temperature dropped to room temperature.

4.3. Preparation of PCN@TA/PEI. Tannic acid/polyethyleneimine (TA/PEI) nanoshell was prepared according to our previous study [25]. Firstly, the mixed buffer solution ( $\mathrm{pH}$ 6.0) containing $50 \mathrm{mM}$ Tris- $\mathrm{HCl}$ and $0.5 \mathrm{mg} \mathrm{mL}^{-1} \mathrm{TA}$ was prepared. The PCN ( $80 \mathrm{mg}$ ) as synthesized above was added to it with vigorous stirring for $1 \mathrm{~min}$. Then, different concentrations of PEI $\left(0.5 \mathrm{mg} \mathrm{mL}^{-1}, 5.0 \mathrm{mg} \mathrm{mL}^{-1}\right.$, and $\left.10.0 \mathrm{mg} \mathrm{mL}^{-1}\right)$ were mixed with above solution. The $\mathrm{pH}$ was further adjusted to 8.0, and the mixed solution was stirred for $1 \mathrm{~h}$. Finally, the PCN@TA/PEI was obtained after centrifugation and washing for three times with water.

4.4. Preparation of PCN@TA/PEI-Rh. Firstly, the mixed buffer solution ( $\mathrm{pH}$ 7.0) containing $50 \mathrm{mM}$ phosphatebuffered saline (PBS) and $10 \mathrm{mM}$ 2,2' -bipyridine-5, $5^{\prime}$ dicarboxylic acid was prepared. The PCN@TA/PEI as prepared above was added to it with stirring. Next, EDC and NHS were added to the above solution with both final concentrations of $10 \mathrm{mM}$. After the $\mathrm{pH}$ was adjusted to 7.0 , the mixed solution was stirred for $48 \mathrm{~h}$. The precipitate was obtained, centrifuged at $6000 \mathrm{rpm}$, and washed for three times with water. Then, the previously collected precipitate was added to mixed solution ( $\mathrm{pH}$ 7.0) with shaking for $24 \mathrm{~h}$, which contained $50 \mathrm{mM}$ PBS buffer and $1 \mathrm{mM}\left(\mathrm{Cp} * \mathrm{RhCl}_{2}\right)_{2}$. Finally, the PCN@TA/PEI-Rh was obtained, centrifuged, and washed three times with water.

4.5. Preparation of $\mathrm{SiO}_{2} @ T A / P E I$. Firstly, the mixed buffer solution ( $\mathrm{pH}$ 6.0) containing $50 \mathrm{mM}$ Tris- $\mathrm{HCl}$ and $0.5 \mathrm{mg} \mathrm{mL}^{-1} \mathrm{TA}$ was prepared. The $\mathrm{SiO}_{2}(80 \mathrm{mg})$ was added to it with vigorous stirring for $1 \mathrm{~min}$. Then, $5.0 \mathrm{mg} \mathrm{mL}^{-1} \mathrm{PEI}$ was mixed with above solution. The $\mathrm{pH}$ was further adjusted to 8.0 , and the mixed solution was stirred for $1 \mathrm{~h}$. Finally, the $\mathrm{SiO}_{2} @ \mathrm{TA} / \mathrm{PEI}$ was obtained after centrifugation and washing for three times with water.

4.6. Synthesis of $\left[\mathrm{Cp} * \mathrm{Rh}(b p y) \mathrm{H}_{2} \mathrm{O}\right]^{2+}$. Pentamethylcyclopentadienyl rhodium bipyridine $\left(\left[\mathrm{Cp} * \mathrm{Rh}(\mathrm{bpy})\left(\mathrm{H}_{2} \mathrm{O}\right)\right]^{2+}\right)$ was prepared according to previous literature [30]. Firstly, $61.8 \mathrm{mg}$ of $\left(\mathrm{Cp} * \mathrm{RhCl}_{2}\right)_{2}$ was added into methanol $(4 \mathrm{~mL})$ to obtain a red suspension. Then, $31.2 \mathrm{mg}$ of 2,2-bipyridine was mixed with the suspension, acquiring a transparent orange solution. At room temperature, after concentrating the solution to $1 \mathrm{~mL}$, anhydrous ether $(8 \mathrm{~mL})$ was added so that $[\mathrm{Cp} * \mathrm{Rh}(\mathrm{bpy}) \mathrm{Cl}] \mathrm{Cl}$ gradually precipitated out. The $[\mathrm{Cp} * \mathrm{Rh}(\mathrm{bpy}) \mathrm{Cl}] \mathrm{Cl}$ was obtained followed by centrifugation and washing for three times with water. After drying in oven, $[\mathrm{Cp} * \mathrm{Rh}($ bpy $) \mathrm{Cl}] \mathrm{Cl}$ was dissolved in $\mathrm{H}_{2} \mathrm{O}$ to obtain [Cp* $\mathrm{Rh}$ (bpy) $\left.\mathrm{H}_{2} \mathrm{O}\right]^{2+}$ (denoted as $\mathrm{Rh}$ ), which was stored at $4^{\circ} \mathrm{C}$ in darkness and utilized to catalyze reaction of $\mathrm{NADH}$ regeneration.
4.7. Photoelectrochemical Measurement. All photoelectrochemical measurements were performed by an electrochemical workstation (CHI660) with a three-electrode system. Typically, the reference electrode was $\mathrm{Ag} / \mathrm{AgCl}$, the counter electrode was platinum foil $\left(0.5 \times 0.5 \mathrm{~cm}^{2}\right)$, and the prepared samples were used as the working electrodes. The working electrodes were prepared by dispersing samples $(5 \mathrm{mg})$ into $2 \%$ Nafion solution $(0.5 \mathrm{~mL})$ and dropping it onto FTO glass electrode $\left(2.0 \times 1.5 \mathrm{~cm}^{2}\right)$. After drying overnight in $40^{\circ} \mathrm{C}$ oven, the films on FTO glass electrode were divided into $1.0 \times 1.5 \mathrm{~cm}^{2}$ for subsequent measurement. The $0.1 \mathrm{M}$ $\mathrm{Na}_{2} \mathrm{SO}_{4}$ was used as electrolyte, and the light source was a $300 \mathrm{~W}$ xenon lamp. The initial voltage of the transient photocurrent and electrochemical impedance spectroscopy (EIS) was kept at $-0.1 \mathrm{~V}$.

4.8. Photocatalytic Regeneration of NADH. The photocatalytic NADH regeneration was proceeded in a quartz reactor $\left(1.25 \times 1.25 \times 4.5 \mathrm{~cm}^{3}\right)$ with reaction mixture $(2 \mathrm{~mL})$, which contained $0.5 \mathrm{mg} \mathrm{mL}^{-1}$ photocatalyst, $1 \mathrm{mM} \mathrm{NAD}^{+}, 400 \mathrm{mM}$ TEOA, and $100 \mathrm{mM}$ PBS $(\mathrm{pH}=8.0)$. Firstly, the mixture was hatched for $10 \mathrm{~min}$ without illumination, and then a $100 \mathrm{~W}$ LED lamp ( $405 \mathrm{~nm}$ ) was used to activate photocatalyst for $24 \mathrm{~min}$. The sample was taken every $4 \mathrm{~min}$, of which the absorbance at $340 \mathrm{~nm}$ was detected by a UV-vis spectrophotometer (U-3010, Hitachi). On the basis of the detected absorbance with the following equation, we could calculate the concentration of regenerated $\mathrm{NADH}$.

$$
c=\frac{A}{K b},
$$

where $c$ was the concentration of regenerated NADH, $A$ was the detected absorbance at $340 \mathrm{~nm}$ of sample, $K$ was an extinction coefficient of $6220 \mathrm{M}^{-1} \mathrm{~cm}^{-1}$ [26], and $b$ was the optical path of $1 \mathrm{~cm}$.

The turnover frequency (TOF) of NADH based on Rh was calculated with the following equation:

$$
\mathrm{TOF}=\frac{c_{\mathrm{NADH}}}{c_{\mathrm{Rh}} t},
$$

where $c_{\mathrm{NADH}}$ and $c_{\mathrm{Rh}}$ were the concentrations of regenerated $\mathrm{NADH}$ and $\left[\mathrm{Cp} * \mathrm{Rh}(\text { bpy }) \mathrm{H}_{2} \mathrm{O}\right]^{2+}(\mathrm{Rh})$, respectively and $t$ was $4 \mathrm{~min}$ (based on the $4-8 \mathrm{~min}$ of reaction).

4.9. Photoenzymatic Production of Formate from $\mathrm{CO}_{2}$. Photoenzymatic conversion of $\mathrm{CO}_{2}(0.3 \mathrm{MPa})$ was performed in a visible photocatalytic autoclave with $4 \mathrm{~mL}$ mixed suspension $\left(37^{\circ} \mathrm{C}\right)$, which contained $0.5 \mathrm{mg} \mathrm{mL}^{-1}$ photocatalyst, $100 \mathrm{mM}$ PBS $(\mathrm{pH}=8.0), 5 \mathrm{mM} \mathrm{NAD}{ }^{+}, 400 \mathrm{mM}$ TEOA, and $2 \mathrm{mg} \mathrm{mL}^{-1}$ formate dehydrogenase (FDH). A $100 \mathrm{~W}$ LED lamp (405 nm) was used as optical source for $120 \mathrm{~min}$. After every $10 \mathrm{~min}$ illumination, the sample was taken and centrifuged. The concentration of formic acid in the supernatant was detected by previously reported chromogenic method [31].

According to the enzymatic reaction $\mathrm{CO}_{2}+\mathrm{NADH} \stackrel{\mathrm{FDH}}{\longrightarrow}$ $\mathrm{HCOO}^{-}+\mathrm{NAD}^{+}, \mathrm{NADH}$ was consumed following the 
stoichiometric ratio during the reaction of formate production. The mole of regenerated $\mathrm{NADH}$ was the same as that of formate produced during the reaction. The times of individual NADH utilized in formate production were calculated with the following equation:

$$
N_{\mathrm{NADH}}=\frac{c_{\text {Formate }}}{c_{\mathrm{NADH}}},
$$

where $N_{\mathrm{NADH}}$ was the times of individual NADH used for formate production, $c_{\text {Formate }}$ was the concentration of formate, and $c_{\mathrm{NADH}}$ was the concentration of NADH.

4.10. Characterizations. Fourier transform infrared (FTIR) spectra were conducted by a Nicolet-6700 spectrometer. $\mathrm{X}$-ray powder diffraction (XRD) was collected on a Rigaku $\mathrm{D} / \max 2500 \mathrm{~V} / \mathrm{PC}$ instrument, and the data was obtained in the range of $10-60^{\circ}(2 \theta)$ at a rate of $5^{\circ} \mathrm{min}^{-1}$. Transmission electron microscopy (TEM) images were measured on a JEM-2100F instrument. Elemental analysis was performed in elemental mappings attached to transmission electron microscopy (TEM). X-ray photoelectron spectroscopy (XPS) was recorded on a PerkinElmer PHI 1600 ESCA spectroscope with monochromatic $\mathrm{Mg} \mathrm{K} \alpha$ radiation. UV-vis absorption spectra were conducted by a Hitachi U3010 spectrometer. Photoluminescence (PL) spectra were recorded on a Jobin Yvon Fluorolog-3 fluorescence spectrometer with excitation at $350 \mathrm{~nm}$.

\section{Data Availability}

All data used to support the findings of this study are available from the corresponding author upon reasonable request.

\section{Conflicts of Interest}

The authors declare no conflict of interest.

\section{Acknowledgments}

This work was supported by the National Natural Science Funds of China (21776213 and 21621004), the Natural Science Fund of Tianjin (19JCYBJC19700), the Open Funding Project of the State Key Laboratory of Biochemical Engineering (2020KF-06), the Open Funding Project of the State Key Laboratory of Chemical Engineering (SKL-ChE19B01), the Tianjin Synthetic Biotechnology Innovation Capacity Improvement Project (TSBICIP-KJGG-003), and the Royal Society International Collaboration Award (ICA $\backslash \mathrm{R} 1 \backslash 180317)$ for the financial support. The National Supercomputing Center in Shenzhen is acknowledged for the computational support.

\section{Supplementary Materials}

Figure S1: schematic reaction mechanism during the preparation of PCN@TA/PEI-Rh core@shell photocatalyst. Figure S2: TEM images of PCN@TA/PEI-Rh (a) and corresponding element mapping of (b) C, (c) N, (d) O, and (e) Rh of PCN@TA/PEI-Rh. Figure S3: XRD patterns of PCN,
PCN@TA/PEI, and PCN@TA/PEI-Rh. Figure S4: FT-IR spectra of PCN, PCN@TA/PEI, and PCN@TA/PEI-Rh. Figure S5: high-resolution XPS C 1s spectra of PCN, PCN@TA/PEI, and PCN@TA/PEI-Rh. Figure S6: highresolution XPS O 1s spectra of PCN, PCN@TA/PEI, and PCN@TA/PEI-Rh. Figure S7: UV-vis diffuse reflectance spectra of PCN, PCN@TA/PEI, and PCN@TA/PEI-Rh. Figure S8: photocurrent responses of PCN and PCN@TA/PEIRh at $-0.1 \mathrm{~V}$ vs. Ag/AgCl. Figure S9: PL spectra of PCN and PCN@TA/PEI-Rh. Figure S10: configuration of (a) bpy-H and (b) bpy-Rh-H. Figure S11: the concentrations of immobilized $\mathrm{Rh}$ in different $\mathrm{TA}$ and PEI concentration ratios ([TA]/[PEI]). Figure S12: the NADH regeneration efficiency in different coupling reaction time of PEI and bpy-COOH $(X)$. Figure S13: the reaction setup of photocatalytic NADH regeneration. Figure S14: recycle stabilities of PCN@TA/ PEI-Rh in TEOA solution. Table S1: parameters of timeresolved transient PL described by biexponential function. Table S2: the photocatalytic regeneration performance of NADH by different photocatalysts. Table S3: the experimental data and $r_{\mathrm{NADH}}$ for photocatalytic NADH regeneration with PCN and PCN@TA/PEI-Rh. (Supplementary Materials)

\section{References}

[1] Z. C. Litman, Y. Wang, H. Zhao, and J. F. Hartwig, "Cooperative asymmetric reactions combining photocatalysis and enzymatic catalysis," Nature, vol. 560, no. 7718, pp. 355359, 2018.

[2] X. Fang, S. Kalathil, and E. Reisner, "Semi-biological approaches to solar-to-chemical conversion," Chemical Society Reviews, vol. 49, no. 14, pp. 4926-4952, 2020.

[3] F. Gu, Y. Wang, Z. Meng, W. F. Liu, and L. Y. Qiu, "A coupled photocatalytic/enzymatic system for sustainable conversion of $\mathrm{CO}_{2}$ to formate," Catalysis Communications, vol. 136, article 105903, 2020.

[4] Y. Chen, P. Li, J. Zhou et al., "Integration of enzymes and photosensitizers in a hierarchical mesoporous metal-organic framework for light-driven $\mathrm{CO}_{2}$ reduction," Journal of the American Chemical Society, vol. 142, no. 4, pp. 1768-1773, 2020.

[5] K. A. Brown, Q. Song, D. W. Mulder, and P. W. King, "Diameter dependent electron transfer kinetics in semiconductorenzyme complexes," ACS Nano, vol. 8, no. 10, pp. 10790 10798, 2014.

[6] M. Karayilan, W. P. Brezinski, K. E. Clary, D. L. Lichtenberger, R. S. Glass, and J. Pyun, "Catalytic metallopolymers from [2Fe-2S] clusters: artificial metalloenzymes for hydrogen production," Angewandte Chemie International Edition, vol. 58, no. 23, pp. 7537-7550, 2019.

[7] C. Tapia, S. Shleev, J. C. Conesa, A. L. de Lacey, and M. Pita, "Laccase-catalyzed bioelectrochemical oxidation of water assisted with visible light," ACS Catalysis, vol. 7, no. 7, pp. 4881-4889, 2017.

[8] S. Roy, V. Jain, R. K. Kashyap, A. Rao, and P. P. Pillai, "Electrostatically driven multielectron transfer for the photocatalytic regeneration of nicotinamide cofactor," ACS Catalysis, vol. 10, no. 10, pp. 5522-5528, 2020.

[9] H. Zhang, Y. Zhao, H. Zhang et al., "Establishing inorganicbiological hybrid photoelectrochemical platform towards 
sustainable conversion of A-chitin," Applied Catalysis B: Environmental, vol. 265, article 118558, 2020.

[10] A. R. Oliveira, C. Mota, C. Mourato et al., "Toward the mechanistic understanding of enzymatic CO2 Reduction," ACS Catalysis, vol. 10, no. 6, pp. 3844-3856, 2020.

[11] S. H. Lee, D. S. Choi, S. K. Kuk, and C. B. Park, "photobiocatalysis: activating redox enzymes by direct or indirect transfer of photoinduced electrons," Angewandte Chemie International Edition, vol. 57, no. 27, pp. 7958-7985, 2018.

[12] J. H. Kim, M. Lee, J. S. Lee, and C. B. Park, "Self-assembled light-harvesting peptide nanotubes for mimicking natural photosynthesis," Angewandte Chemie International Edition, vol. 51, no. 2, pp. 517-520, 2012.

[13] Z. W. Tong, D. Yang, Z. Li et al., "Thylakoid-inspired multishell g-C3N4 Nanocapsules with enhanced visible-light harvesting and electron transfer properties for highefficiency photocatalysis," ACS Nano, vol. 11, no. 1, pp. 1103-1112, 2017.

[14] V. S. Vyas, F. Haase, L. Stegbauer et al., "A tunable azine covalent organic framework platform for visible light- induced hydrogen generation," Nature Communications, vol. 6, no. 1, article 8508, 2015.

[15] Y. Z. Wu, J. Ward-Bond, D. Li, S. Zhang, J. Shi, and Z. Jiang, "g- $\mathrm{C}_{3} \mathrm{~N}_{4} @ \alpha-\mathrm{Fe}_{2} \mathrm{O}_{3} / \mathrm{C}$ photocatalysts: synergistically intensified charge generation and charge transfer for NADH regeneration," ACS Catalysis, vol. 8, no. 7, pp. 5664-5674, 2018.

[16] L. Q. Kong, J. Q. Yan, P. Li, and S. F. Liu, " $\mathrm{Fe}_{2} \mathrm{O}_{3} / \mathrm{C}-\mathrm{C}_{3} \mathrm{~N}_{4}$ based tight heterojunction for boosting visible-light-driven photocatalytic water oxidation," ACS Sustainable Chemistry \& Engineering, vol. 6, no. 8, pp. 10436-10444, 2018.

[17] D. Yang, Y. S. Zhang, S. H. Zhang et al., "Coordination between electron transfer and molecule diffusion through a bioinspired amorphous titania nanoshell for photocatalytic nicotinamide cofactor regeneration," ACS Catalysis, vol. 9, no. 12, pp. 11492-11501, 2019.

[18] F. M. Zhang, J. L. Sheng, Z. D. Yang et al., "Rational design of MOF/COF hybrid materials for photocatalytic H2 Evolution in the presence of sacrificial electron donors," Angewandte Chemie International Edition, vol. 57, no. 37, pp. 1210612110, 2018.

[19] K. T. Oppelt, J. Gasiorowski, D. A. Egbe et al., "Rhodium-coordinated poly(arylene-ethynylene)-alt-poly(arylene-vinylene) copolymer acting as photocatalyst for visible-light-powered $\mathrm{NAD}^{+} / \mathrm{NADH}$ reduction," Journal of the American Chemical Society, vol. 136, no. 36, pp. 12721-12729, 2014.

[20] J. F. Allen, W. B. M. de Paula, S. Puthiyaveetil, and J. Nield, "A structural phylogenetic map for chloroplast photosynthesis," Trends in Plant Science, vol. 16, no. 12, pp. 645-655, 2011.

[21] N. Nelson, "Plant photosystem I - the most efficient nanophotochemical machine," Journal of Nanoscience Nanotechnology, vol. 9, no. 3, pp. 1709-1713, 2009.

[22] X. C. Qin, M. Suga, T. Y. Kuang, and J. R. Shen, "Structural basis for energy transfer pathways in the plant PSI-LHCI supercomplex," Science, vol. 348, no. 6238, pp. 989-995, 2015.

[23] J. J. Wilker, "Positive charges and underwater adhesion," Science, vol. 349, no. 6248, pp. 582-583, 2015.

[24] T. Priemel, E. Degtyar, M. N. Dean, and M. J. Harrington, "Rapid self-assembly of complex biomolecular architectures during mussel byssus biofabrication," Nature Communications, vol. 8, no. 1, p. 12, 2017.
[25] Z. Cai, J. Shi, W. Li et al., "Mussel-inspired pH-switched assembly of capsules with an ultrathin and robust nanoshell," ACS Applied Materials \& Interfaces, vol. 11, no. 31, pp. 28228-28235, 2019.

[26] J. Liu and M. Antonietti, "Bio-inspired NADH regeneration by carbon nitride photocatalysis using diatom templates," Energy \& Environmental Science, vol. 6, no. 5, pp. 1486-1493, 2013.

[27] X. D. Wang, T. Saba, H. H. P. Yiu, R. F. Howe, J. A. Anderson, and J. Shi, "Cofactor NAD $(\mathrm{P}) \mathrm{H}$ regeneration inspired by heterogeneous pathways," Chem, vol. 2, no. 5, pp. 621-654, 2017.

[28] L. Tensi and A. Macchioni, "Extremely fast NADHregeneration using phosphonic acid as hydride source and iridium-pyridine-2-sulfonamidate catalysts," ACS Catalysis, vol. 10, no. 14, pp. 7945-7949, 2020.

[29] S. Zhang, J. Shi, Y. Sun et al., "Artificial thylakoid for the coordinated photoenzymatic reduction of carbon dioxide," ACS Catalysis, vol. 9, no. 5, pp. 3913-3925, 2019.

[30] F. Hollmann, B. Witholt, and A. Schmid, "[Cp* $\mathrm{Rh}(\mathrm{bpy})(\mathrm{H} 2 \mathrm{O})]^{2+}$ : a versatile tool for efficient and nonenzymatic regeneration of nicotinamide and flavin coenzymes," Journal of Molecular Catalysis B-Enzymatic, vol. 19, pp. 167-176, 2002.

[31] R. Sleat and R. A. Mah, "Quantitative method for colorimetric determination of formate in fermentation media," Applied and Environmental Microbiology, vol. 47, no. 4, pp. 884-885, 1984. 